Notfall Rettungsmed 2011 1 14:257-258

DOI 10.1007/s10049-011-1470-8

Online publiziert: 26. Mai 2011

(c) Springer-Verlag 2011

\author{
H.-R. Arntz ${ }^{1}$ • U. Kreimeier ${ }^{2}$ \\ ${ }^{1}$ Medizinische Klinik II, Kardiologie und Pulmonologie, \\ Charité - Universitätsmedizin Berlin, Campus Benjamin Franklin, Berlin \\ ${ }^{2}$ Klinik für Anaesthesiologie, Klinikum der Universität München, München
}

\title{
Erfolgreiches Risikomanagement in der Katastrophenmedizin
}

Nach allgemeiner Definition sind Katastrophen Großschadenereignisse, zu deren Bewältigung die regionalen personellen, technischen und materiellen Resourcen nicht ausreichen. Wir leben in einer Welt, in der Katastrophen gelegentlich wie in einer nicht enden wollenden Kette von Geschehnissen aufeinanderfolgen. Zweifellos ist die Nähe und unmittelbare Betroffenheit, die wir dabei erleben, auch Folge der innerhalb von Minuten verfügbaren Bilder vom Geschehen.

Die Ursachen der katastrophalen Ereignisse sind vielfältig und leider oft auch vom leichtfertigen Wirken des Menschen beeinflusst oder sogar verursacht. Ein krasses Beispiel der Folgen menschlichen Fehlverhaltens ist die Explosion im Reaktorblock 4 in Tschernobyl, das sich gerade zum 25. Mal jährt. In einem Testlauf zur Simulation eines totalen Stromausfalls führte die Kombination von Missachtung von Sicherheitsvorschriften mit technischen Besonderheiten zur Explosion des Reaktors und dem atomaren GAU, in dessen Folge riesige Mengen Radioaktivität freigesetzt wurden und sich über Europa ausbreiteten. Bis heute sind die Konsequenzen weder hinsichtlich der Zahl der Opfer noch der bis heute weiterreichenden Strahlenrisiken mit auch zukünftigen Opfern noch hinsichtlich der wirtschaftlichen und finanziellen Folgen sowie der psychologischen und sozialen Schäden bezifferbar. Erdbeben, der Tsunami in Indonesien, Vulkanausbrüche, Schlammlawinen und Überschwemmungen sind „natürliche“ Katastrophen mit unermesslicher Zerstörungsgewalt und potenziell Hunderttausenden von Opfern. Die Auswirkungen dieser Ereig- nisse sind allerdings auch teilweise durch menschliches Zutun verschlimmert worden, sei es durch ungenügende Schutzmaßnahmen, gravierende Baumängel, im schlimmsten Fall bewusst in Kauf genommen, oder auch durch leichtfertige Besiedlung von Risikoarealen. Besonders gravierend sind die Auswirkungen, wenn Naturkatastrophen technische Katastrophen verursachen oder mit ihnen zusammenfallen, wie uns Fukushima vor Augen führt. Das Tschernobylexperiment „totaler Stromausfall“ ist in Fukushima in tragischer Weise durch die Auswirkungen des Tsunami in die Realität mit noch nicht absehbaren Folgen katastrophalen Ausmaßes umgesetzt worden und hat uns die Bedeutung des vorgeblich extrem unwahrscheinlichen, dafür aber um so fataleren unkalkulierbaren „Restrisikos“ dramatisch vor Augen geführt.

Manch einer mag denken, Tschernobyl und Fukushima sind weit entfernt. Es sei jedoch daran erinnert, dass $\mathrm{Na}$ turkatastrophen wie z. B. die Hamburger Sturmflut von 1962, das Oder- und Elbehochwasser 2002 und das Elbehochwasser 2006 auch in Deutschland passiert sind und damit potenziell auch hier drohen. Auch Schlampereien und Leitsinnigkeit haben zu schweren Unglücken geführt, so z. B. die mangelnde Radreifeninspektion, die zum Unglück des ICE Konrad Röntgen in Eschede führte, oder die jahrelange mangelnde Beaufsichtigung oder sogar unkontrollierte Einlagerung von radiaktivem Abfall und möglicherweise hochtoxischem Sondermüll in die jetzt vom Einsturz bedrohte „Asse“, deren sichere Entsorgung Milliarden kosten wird und hoffentlich keine weiteren Folgen hat. Orga- nisationsmängel und unvorhergesehene Abläufe können auch geplante Großveranstaltungen zur Katastrophe werden lassen, siehe die Loveparade 2010 in Duisburg. Ende April wurde bekannt, dass auf dem PC des versuchten Terroranschlags auf dem Times Square in New York Hinweise für einen möglicherweise geplanten Terroranschlag in der Stuttgarter Innenstadt gefunden wurden. Eine weltweit operierendes Terrornetzwerk stellt ein permanentes Gefährdungspotenzial dar.

Der konventionelle Katastrophenbegriff erfährt in unserer rettungsdienstlichen Tätigkeit noch eine weitere Dimension, und zwar im persönlichen Bereich: Der unerwartete plötzliche Tod an einer akuten Erkrankung, der Unfalltod auf der Autobahn oder am Arbeitsplatz wird von den Angehörigen zu Recht und häufig auch von uns im Notfalldienst als Katastrophe empfunden, die nicht selten zu ihrer Bewältigung äußerer Hilfe Bedarf. So verstanden kann unsere alltägliche Notfallmedizin ebenfalls als eine Art fokussierte Katastrophenmedizin verstanden werden, wenn auch eben nur „im Kleinen“, wobei wir versuchen müssen zu vermeiden, die Dinge erst durch unser Handeln zur Katastrophe zu machen.

\section{( Die alltägliche Notfallmedizin kann als fokussierte Katastrophenmedizin verstanden werden}

Als Mitarbeiter in der Notfallmedizin ist man häufig zu sofortigem, zumindest jedoch schnellem Handeln unter oft widrigen äußeren Umständen gezwungen. Unweigerlich sind damit besondere Ri- 
siken verbunden. Diese Risiken so gering wie möglich zu halten ist berechtigter Anspruch unserer Patienten und verlangt vom Notfallteam auf der Straße oder in der Notfallambulanz ein besonderes Risikobewusstsein und demzufolge sowohl ein fundiertes theoretisches Wissen als auch eingeübte technische Fertigkeiten. Ein angemessenes Risikomanagement hilft vermeidbare Risiken in den Griff zu bekommen und damit fatalen Verläufen vorzubeugen. Zum Risikomanagement eignen sich eine Vielzahl von Hilfsmitteln und Lehrmethoden, sei es in Form von Check-Listen, Team-Übungen, FallSimulationen, technischem Training und vielem anderem mehr. Hoffen wir, dass wir keine großen Katastrophen erleben, versuchen wir, durch ein eigenes Risikomanagement Katastrophen zu Lasten unserer Patienten auch ,im Kleinen“ zu vermeiden, und seien wir uns bewusst, dass trotzdem bei jedem Einsatz immer ein unkalkulierbares Restrisiko bleibt, dessen Eintritt zu vermeiden immer im Auge behalten werden muss.

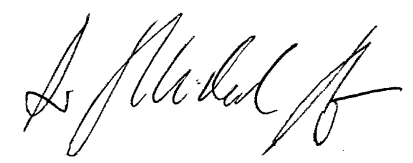

H.-R. Arntz

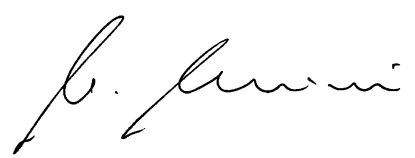

U. Kreimeier

\section{Korrespondenzadresse}

\section{Prof. Dr. H.-R. Arntz}

Medizinische Klinik II, Kardiologie und Pulmonologie,

Charité - Universitätsmedizin Berlin, Campus Benjamin Franklin Hindenburgdamm 30, 12200 Berlin Hans-Richard.Arntz@charite.de
W. Meißner

\section{Akutschmerz Taschenbuch}

Berlin: Medizinisch wissenschaftliche Verlagsgesellschaft 2011, 250 S., 50 Abb., 30 Tab., brosch., 29.00 EUR

Die Literaturlandschaft rund um das Thema Schmerzmedizin ist um einen wertvollen Ratgeber im Kitteltaschenformat erweitert worden.

Das Buch, konzipiert unter der Federführung von PD Winfried Meißner, Mitglied in der Kommission für Aus-,Weiter- und Fortbildung der DGSS und 2. Sprecher des Arbeitskreises Schmerzmedizin der DGAl, spricht mit seinem übersichtlichen Aufbau in 15 kurz gefassten Kapiteln alle relevanten Themen der perioperativen Akutschmerztherapie auf leicht verständliche einprägsame Weise an. Zielgruppe sollen dabei nicht nur die mit der Aufgabe "Schmerztherapie" betrauten oft überlasteten StationsärztInnen bzw. OP-AnästhesistInnen sein, sondern ebenso an der Schmerztherapie beteiligte Pflegekräfte, Physiotherapeuten, Psychologen und, sofern als Organisationsform bereits vorhanden, hauptberufliche Mitarbeiter eines Akutschmerzdienstes im Krankenhaus. Zur schnellen Orientierung im täglichen Gebrauch tragen mehrere Elemente bei. Der Inhalt fokussiert auf die konkrete Anwendung theoretischen Wissens in alltäglichen klinischen Szenarien, ohne sich in unnötigen Details zu verlieren. Dabei gelingt es gleichzeitig, an den richtigen Stellen Querverweise auf die zugrundeliegende Evidenz oder auf weiterführende Literatur einzuflechten. Darüber hinaus dienen Texthervorhebungen in Form von Merksätzen, Praxistipps oder Hintergrundinformationen in überschaubarer Anzahl und Verteilung einer konzentrierten Informationsvermittlung. Zusammenfassungen in Stichpunkten am Ende der Kapitel runden das didaktische Konzept sinnvoll ab. Die ersten beiden Kapitel befassen sich zunächst mit den Rahmenbedingungen in der perioperativen Schmerztherapie. Behandelt werden sowohl mögliche Organistionsformen einer strukturierten Schmerztherapie als auch die logistischen Anforderungen, die sich aus der Vielfalt moderner chirurgischer Behandlungsmethoden (z.B. "Fast-Track-Chirurgie") in den verschiede- nen Disziplinen ergeben. Es folgen, einer redundanten Systematik folgend, Kapitel zu pharmakologischen Aspekten. Dabei werden alle Bereiche, von den zur Verfügung stehenden Medikamenten mit deren typischen Anwendungsbereichen und zu erwartenden Nebenwirkungen über spezifische Behandlungsmethoden, wie die „patienten-kontrollierte Analgesie“ unter Zuhilfenahme programmierbarer Pumpensysteme, bis hin zu bewährten Therapiestandards bzw. Beispielen für "Standard Operating Procedures" in der postoperativen Schmerztherapie vorgestellt. Hier gefällt die sehr praxisnahe Hilfestellung mit direkt anwendbaren Musterlösungen, die als Basiskonzept auf viele Versorgungseinheiten übertragen werden können. In der zweiten Hälfte des Taschenbuches wird als folgerichtige Erweiterung zu den „basics" fundiertem Spezialwissen zu ausgewählten Themen, die in der täglichen Arbeit eines Akutschmerzdienstes einen festen Platz einnehmen, angemessener Raum gegeben. Neben nichtmedikamentösen Verfahren, wie Physiotherapie, Akupunktur oder transkutane elektrische Nervenstimulation (TENS), als sinnvolle Konzeptvervollständigung werden auch Handlungsstrategien bei postoperativer Übelkeit und Erbrechen (PONV), sowie Therapiekonzepte zur systematischen Einbeziehung von Patienten im Kindesalter und geriatrischen Patienten in eine standardisierte Akutschmerztherapie aufgezeigt. Auch hier bleibt ein "roter Faden“, der eine unmittelbare Anwendbarkeit in der Klinik ermöglicht, trotz der Komplexität dieser Themengebiete klar erkennbar. Eine kurze Darstellung prozedurenspezifischer Maßnahmen gemäß den aktuellen S3-Leitlinien vervollständigt in einer kritischen Gegenüberstellung von Ideallösungen und Limitierungen im OP-Alltag den schmerztherapeutischen Handlungsrahmen. Das Buch liefert schließlich in den letzten beiden Kapiteln wichtige Instrumente der Qualitätssicherung in der Schmerztherapie.

Dr. Jörg Filitz, Prof. Dr. Wolfgang Koppert, Hannover 